\title{
CYCLIC HOMOLOGY FOR SCHEMES
}

\author{
CHARLES WEIBEL \\ (Communicated by Eric Friedlander)
}

\begin{abstract}
Using hypercohomology, we can extend cyclic homology from algebras to all schemes over a ring $k$. By 'extend' we mean that the usual cyclic homology of any commutative algebra agrees with the cyclic homology of its corresponding affine scheme.
\end{abstract}

The purpose of this paper is to show that there is a cyclic homology theory $\mathbb{H} C_{*}$ of schemes over a commutative ring $k$, extending the usual cyclic homology $H C_{*}$ of $k$-algebras. By a cyclic homology theory for schemes over $k$ we mean a family of graded $k$-modules $\mathbb{H} C_{n}(X)$ associated to every scheme $X$ over $k$ which satisfy:

(0.1) they are natural and contravariant in $X$;

(0.2) for each affine scheme $X=\operatorname{Spec} A$, there are natural isomorphisms

$$
\mathbb{H} C_{n}(X) \cong H C_{n}(A) \quad \text { for all } n ;
$$

(0.3) if $X=U \cup V$, there is a Mayer-Vietoris sequence

$$
\cdots \mathbb{H} C_{n}(X) \rightarrow \mathbb{H} C_{n}(U) \oplus \mathbb{H} C_{n}(V) \rightarrow \mathbb{H} C_{n}(U \cap V) \rightarrow \mathbb{H} C_{n-1}(X) \cdots
$$

We discuss uniqueness of a cyclic homology theory briefly in Remark 0.5 below.

We have chosen homological indexing because of axiom (0.2), and because cohomological indexing $\left(\mathbb{H} C^{n}=\mathbb{H} C_{-n}\right.$ ) would concentrate the nonzero groups in negative degrees.

The construction is simple: just sheafify Connes' $(b, B)$ double chain complex and take Cartan-Eilenberg hypercohomology (as defined in the appendix). Axioms (0.1) and (0.3) are immediate from generalities about hypercohomology of unbounded complexes. The point of this paper is that axiom (0.2) also holds.

This definition was suggested by Loday in [L1, 3.4], without knowing whether axiom (0.2) held. Motivated by this gap, Geller and I showed in [WG, 4.6.1] that if $A$ is a finite-dimensional noetherian $k$-algebra, then $(0.2)$ holds, i.e., that $H C_{*}(A) \cong$ $\mathbb{H} C_{*}(\operatorname{Spec} A)$.

In the spirit of Grothendieck's seminal letter [GdR], Geller and I also proved in [WG] that sheafifying and taking hypercohomology yields a "Hochschild homology

Received by the editors April 25, 1994 and, in revised form, November 21, 1994.

1991 Mathematics Subject Classification. Primary 19D55; Secondary 18G60, 14 F05.

Key words and phrases. Cyclic homology, schemes, hypercohomology.

The author was partially supported by NSF grants and is grateful to the Mittag-Leffler Institute for providing the environment needed to finish this research.

(C)1996 American Mathematical Society 
theory" for schemes over $k$ (replace $\mathbb{H} C$ by $\mathbb{H} H$ in the above axioms). The key axiom (0.2) for $\mathbb{H} H$ is a consequence of étale descent. Hochschild and cyclic homology of schemes are related by a natural "SBI" sequence [WG, 4.5.1], generalizing the usual SBI sequence for algebras:

$$
\cdots \mathbb{H} C_{n}(X) \stackrel{S}{\longrightarrow} \mathbb{H} C_{n-2}(X) \stackrel{B}{\longrightarrow} \mathbb{H} H_{n-1}(X) \stackrel{I}{\rightarrow} \mathbb{H} C_{n-1}(X) \cdots
$$

Since hypercohomology of unbounded complexes is an ambiguous notion, I have included an appendix to show how the "Cartan-Eilenberg" hypercohomology used in this paper (and in $[\mathrm{CE}][\mathrm{T}],[\mathrm{W}],[\mathrm{WG}]$, etc.) compares with hypercohomology in the sense of derived categories.

Remark 0.5. Suppose that $\mathbb{H} C_{*} \rightarrow \mathbb{H} C_{*}^{\prime}$ is a morphism of cyclic homology theories. Then $\mathbb{H} C_{*}(X) \cong \mathbb{H} C_{*}^{\prime}(X)$ for every quasi-compact, quasi-separated scheme $X$ over $k$. To see this, note that the affine case is axiom (0.2). The case in which $X$ is quasi-compact and separated follows from the Mayer-Vietoris axiom (0.3), using the 5-lemma and induction on the size of an affine cover. Another induction, again on the size of a quasi-affine cover, proves the result whenever $X$ is quasi-compact and quasi-separated.

\section{§1. The Definitions}

Fix a commutative ring $k$, and write $\otimes$ for $\otimes_{k}$. The Hochschild homology $H H_{n}(A)$ of a $k$-algebra $A$ is the homology of the standard Hochschild complex $C_{*}^{h}(A)$ which has $C_{n}^{h}(A)=A^{\otimes n+1}=A \otimes A \otimes \cdots \otimes A$. Let us write $\mathcal{C}_{*}^{h}$ for the sheafification of the corresponding complex of presheaves $U \mapsto C_{*}^{h}\left(\mathcal{O}_{X}(U)\right)$ :

$$
\mathcal{C}_{*}^{h}: \quad \cdots \stackrel{b}{\rightarrow} \mathcal{O}_{X}^{\otimes *+1} \stackrel{b}{\rightarrow} \cdots \stackrel{b}{\rightarrow} \mathcal{O}_{X} \otimes_{k} \mathcal{O}_{X} \stackrel{0}{\rightarrow} \mathcal{O}_{X} \rightarrow 0 .
$$

As in [WG], [W1], the Hochschild homology of $X$ over $k$ is defined as the (CartanEilenberg) hypercohomology (see the appendix) of the unbounded cochain complex $\mathcal{C}^{n}=\mathcal{C}_{-n}^{h}$ :

$$
\mathbb{H} H_{n}(X)=\mathbb{H}^{-n}\left(X, \mathcal{C}_{*}^{h}\right)=\mathbb{H}^{-n}\left(X, \mathcal{C}^{-*}\right) .
$$

For an affine scheme $X=\operatorname{Spec} A, \mathbb{H} H_{n}(X)$ agrees with the classical group $H H_{n}(A)$ by [WG, 4.1]. Note that $X$ can have negative Hochschild homology modules (viz. $H^{n}\left(X, \mathcal{O}_{X}\right)$ is a summand of $\left.\mathbb{H} H_{-n}(X)\right)$. However, when $X$ is quasi-compact and quasi-separated, the argument of 0.5 shows that only finitely many negative Hochschild modules can be nonzero. For example, $\mathbb{H} H_{n}(X)=0$ for $n<-\operatorname{dim}(X)$ whenever $X$ is finite-dimensional and noetherian over $k$ [WG, 4.3].

Although the sheaves $\mathcal{C}_{n}^{h}=\mathcal{O}_{X}^{\otimes n+1}$ are not quasi-coherent for $n>0$, the following proposition, which is a paraphrase of part of [WG, 0.4], states that the chain complex $\mathcal{C}_{*}^{h}$ has quasi-coherent homology. To formulate it, let $\mathcal{H H}_{n}$ denote the $n^{\text {th }}$ sheaf homology of $\mathcal{C}_{*}^{h}$. Since sheafification is an exact functor, it commutes with taking homology. Hence $\mathcal{H H}_{n}$ is also the sheafification of the presheaf $U \mapsto H H_{n}\left(\mathcal{O}_{X}(U)\right)$.

Proposition 1.2 [WG, 0.4]. Let $X$ be a scheme over $k$. Then each $\mathcal{H H}_{n}$ is a quasi-coherent sheaf on $X$. Moreover, on each affine open $U=\operatorname{Spec}(A)$ of $X$ we have natural isomorphisms

$$
H H_{n}(A) \stackrel{\cong}{\rightrightarrows} H^{0}\left(U, \mathcal{H} \mathcal{H}_{n}\right) .
$$


We now define the cyclic homology of a scheme over $k$. Recall that the cyclic homology of $A$ is $H C_{*}(A)=H_{*}\left(\operatorname{Tot} B_{* *}(A)\right)$, where $B_{* *}(A)$ denotes Connes' $(B, b)$ double complex $[\mathrm{C}],[\mathrm{L}],[\mathrm{W}]$. Sheafifying $B_{* *}$ yields a double complex of sheaves, which we write as $\mathcal{B}_{* *}$. Of course, the $\mathcal{O}_{X}^{\otimes n+1}$ form a cyclic object in the category of sheaves, and $\mathcal{B}_{* *}$ is just the associated $(b, B)$ double complex in the category of sheaves. We define

$$
\mathbb{H} C_{n}(X)=\mathbb{H}^{-n}\left(X, \operatorname{Tot} \mathcal{B}_{* *}\right),
$$

where the (product) total chain complex Tot $\mathcal{B}_{* *}$ is regarded as a cochain complex by reindexing in the usual way. We are going to prove that if $X=\operatorname{Spec}(A)$ is affine, then the natural maps $H C_{n}(A) \rightarrow \mathbb{H} C_{n}(X)$ are isomorphisms. The following calculation shows that $\mathbb{H} C_{*}(X)$ is an interesting theory, and indicates how one could reconstruct the Chern character of $[\mathrm{C}]$ from the standpoint of algebraic geometry.

Example 1.4 (Beckmann [B]). Let $X$ be projective $m$-space $\mathbb{P}_{k}^{m}$, and $A$ the ring $k[t] /\left(t^{m+1}\right)$. Then we have

$$
\mathbb{H} H_{*}\left(\mathbb{P}_{k}^{m}\right)=\left\{\begin{array}{l}
A \text { if } *=0, \\
0 \text { if } * \neq 0,
\end{array} \mathbb{H} C_{*}\left(\mathbb{P}_{k}^{m}\right)=H C_{*}(k) \otimes A=\left\{\begin{array}{l}
A \text { if } * \text { is even, } \\
0 \text { if } * \text { is odd. }
\end{array}\right.\right.
$$

The hypercohomology spectral sequence $E_{2}^{p q}=H^{p}\left(X, \mathcal{H H}_{-q}\right) \Rightarrow \mathbb{H} H_{-n}(X)$, which degenerates for $X=\mathbb{P}_{k}^{m}$, gives the calculation for $\mathbb{H} H$. The calculation of $\mathbb{H} C$ follows from this using the SBI sequence (0.4).

Remark 1.5. We can define $\lambda$-operations upon the Hochschild complex $\mathcal{C}_{*}^{h}$ and Connes' double complex $\mathcal{B}_{* *}$. The construction is natural enough that $\lambda$-operations are also defined on $\mathbb{H} H_{*}(X)$ and $\mathbb{H} C_{*}(X)$. If $k$ contains the rational numbers, and if $X$ is noetherian and finite-dimensional, these operations induce $\lambda$-decompositions of the hyperhomology groups, $\mathbb{H} H_{*}(X)=\prod_{i=0}^{\infty} \mathbb{H} H_{*}^{(i)}(X)$ and $\mathbb{H} C_{*}(X)=$ $\prod_{i=0}^{\infty} \mathbb{H} C_{*}^{(i)}(X)$, with

$$
\mathbb{H} H_{n}^{(0)}(X)=\mathbb{H} C_{n}^{(0)}(X)=H^{-n}\left(X, \mathcal{O}_{X}\right) .
$$

We will discuss this decomposition in another paper [W1]. (It is also studied in [B].)

\section{§2. Agreement For AfFine SChemes}

We will make use of the following trick for writing any bounded below chain complex $C_{*}$ as the inverse limit of a natural tower of bounded complexes and surjective maps. The $r^{t h}$ complex in this tower is the "good truncation" $\tau_{<r} C_{*}$ of $C_{*}$ at level $r$ [W, 1.2.7]. By definition, $\tau_{<r} C_{*}$ is the quotient complex of $C_{*}$ such that $\tau_{<r} C_{n}$ is $C_{n}$ when $n<r, d\left(C_{r}\right)$ when $n=r$, and is zero when $n>r$. The adjective "good" refers to the fact that $H_{n}\left(\tau_{<r} C_{*}\right)$ equals $H_{n}\left(C_{*}\right)$ or zero, depending on whether $n<r$ or $n \geq r$. As $r$ varies, the natural surjections $\tau_{<r+1} C_{*} \rightarrow \tau_{<r} C_{*}$ fit together into a tower with inverse limit $C_{*}$. When $C_{*}$ is bounded below, this is a tower of bounded complexes.

In particular, $\mathcal{C}_{*}^{h}$ is the inverse limit of its good truncations $\tau_{<r} \mathcal{C}_{*}^{h}$. We shall write $\mathcal{H H}_{n}\langle r\rangle$ for $H_{n}\left(\tau_{<r} \mathcal{C}_{*}^{h}\right)$; by the above remarks, this equals $H_{n}\left(\mathcal{C}_{*}^{h}\right)=\mathcal{H H}_{n}$ if $n<r$, and equals zero if $n \geq r$. Therefore each $\tau_{<r} \mathcal{C}_{*}^{h}$ is a bounded complex with quasicoherent cohomology. We shall also write $\mathbb{H} H_{n}\langle r\rangle(X)$ for the hypercohomology $\mathbb{H}^{-n}\left(X, \tau_{<r} \mathcal{C}_{*}^{h}\right)$. 
Lemma 2.1. For any affine scheme $X=\operatorname{Spec}(A)$ we have

$$
\mathbb{H} H_{n}\langle r\rangle(X)= \begin{cases}H H_{n}(A) & \text { if } n<r \\ 0 & \text { if } n \geq r .\end{cases}
$$

Proof. Since $\tau_{<r} \mathcal{C}_{*}^{h}$ is a bounded complex, the hypercohomology spectral sequence $E_{2}^{p q}=H^{p}\left(X, H_{-q}\left(\tau_{<r} \mathcal{C}_{*}^{h}\right)\right) \Rightarrow \mathbb{H} H_{-n}\langle r\rangle(X)$ converges. It degenerates, since $\tau_{<r} \mathcal{C}_{*}^{h}$ has quasi-coherent cohomology, to yield $\mathbb{H} H_{n}\langle r\rangle(X)=H^{0}\left(X, \mathcal{H} \mathcal{H}_{n}\langle r\rangle\right)$. Now (1.2) applies.

Definition 2.2. Let $\tau_{q<r} B_{* *}(A)$ denote the double chain complex obtained from Connes' $(b, B)$ double complex $B_{* *}(A)$ by applying the good truncation $\tau_{<r}$ to each column. This truncated double complex is nonzero only in the bounded triangular portion of the plane defined by the equations $0 \leq p \leq q, 0 \leq q \leq r$. The top row of $\tau_{q<r} B_{* *}(A)$ looks like:

$$
0 \leftarrow b\left(A^{\otimes n+1}\right) \stackrel{B}{\longleftarrow} b\left(A^{\otimes n}\right) \stackrel{B}{\longleftarrow} \cdots \stackrel{B}{\longleftarrow} b\left(A^{\otimes 2}\right) \leftarrow 0 .
$$

We define $H C_{n}\langle r\rangle(A)$ to be the homology of Tot $\tau_{q<r} B_{* *}(A)$. Since $\tau_{q<0} B_{* *}(A)=$ $0, H C_{*}\langle 0\rangle(A)=0$. Since $\tau_{q<1} B_{* *}(A)$ is $A$ concentrated in degree zero, the first nonzero truncation is $H C_{0}\langle 1\rangle(A)=A$, with $H C_{n}\langle 1\rangle(A)=0$ for $n \neq 0$.

\section{Lemma 2.3.}

$$
H C_{n}\langle r\rangle(A)= \begin{cases}H C_{n}(A) & \text { if } n<r \\ S\left(H C_{2 r-n}(A)\right) & \text { if } r \leq n \leq 2 r-2, \\ 0 & \text { if } n>2 r-2 .\end{cases}
$$

Proof. This is clear unless $r \leq n \leq 2 r-2$. In general, the inclusion of the first column produces a short exact sequence of chain complexes

$$
0 \rightarrow \tau_{<r} C_{*}^{h}(A) \rightarrow \operatorname{Tot} \tau_{q<r} B_{* *}(A) \stackrel{S}{\longrightarrow} \operatorname{Tot} \tau_{q<r-1} B_{* *}(A)[-2] \rightarrow 0 .
$$

The long exact homology sequence yields $H C_{n}\langle r\rangle(A) \cong H C_{n-2}\langle r-1\rangle(A)$ for $n>r$, and $H C_{r}\langle r\rangle(A) \cong$ image $H C_{r}(A) \stackrel{S}{\longrightarrow} H C_{r-2}(A)$. The result is now straightforward.

We may sheafify the truncations of 2.2 to form the bounded double chain complex of sheaves $\tau_{q<r} \mathcal{B}_{* *}$ on $X$, and define $\mathbb{H} C_{n}\langle r\rangle(X)=\mathbb{H}^{-n}\left(X\right.$, Tot $\left.\tau_{q<r} \mathcal{B}_{* *}\right)$.

Lemma 2.4. For any affine scheme $X=\operatorname{Spec}(A)$, and for all $n$ and $r$, we have

$$
H C_{n}\langle r\rangle(A) \cong \mathbb{H} C_{n}\langle r\rangle(X) .
$$

Proof. For each $n$ we have a short exact sequence of complexes of sheaves on $X$ :

$$
0 \rightarrow \tau_{<r} \mathcal{C}_{*}^{h} \rightarrow \operatorname{Tot} \tau_{q<r} \mathcal{B}_{* *} \stackrel{S}{\longrightarrow} \operatorname{Tot} \tau_{q<r-1} \mathcal{B}_{* *}[-2] \rightarrow 0 .
$$


Taking hypercohomology and comparing to (2.3.1) yields a map of long exact sequences

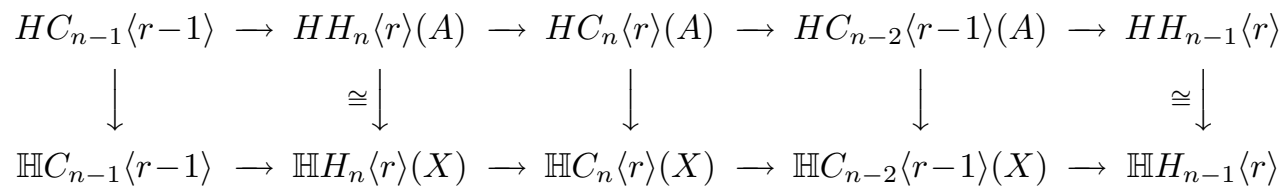

The terms marked ' $\cong$ ' are isomorphisms by Lemma 2.1 . We now proceed by induction on $r$, starting from the trivial case $r=0$.

Remark 2.4.2. The case $r=1, n=0$ of this lemma is the isomorphism $A \cong$ $H^{0}\left(X, \mathcal{O}_{X}\right)$.

Main Theorem 2.5. For any affine scheme $X=\operatorname{Spec}(A)$ over $k$ we have

$$
H C_{n}(A) \cong \mathbb{H} C_{n}(X) \text {. }
$$

Proof. Tot $\mathcal{B}_{* *}$ is the inverse limit of the tower of complexes $\operatorname{Tot}\left(\tau_{q<r} \mathcal{B}_{* *}\right)$, and in each degree $n$ we have $\operatorname{Tot}\left(\mathcal{B}_{* *}\right)_{n}=\operatorname{Tot}\left(\tau_{q<r} \mathcal{B}_{* *}\right)_{n}$ for all $r>n$. Thus we can find Cartan-Eilenberg resolutions $I_{r}^{* *}$ for the $\operatorname{Tot}\left(\tau_{q<r} \mathcal{B}_{* *}\right)$ so that each column $I_{r}^{p *}$ in the tower of double complexes $I_{r}^{* *}$ is eventually constant. By construction, $\lim I_{r}^{* *}$ is a Cartan-Eilenberg resolution of Tot $\mathcal{B}_{* *}$. By (1.3) and (A.1), $\mathbb{H} C_{n}(X)$ is $\overleftarrow{H^{-n}}$ of

$$
\Gamma \operatorname{Tot}\left(\lim _{\longleftarrow} I_{r}^{* *}\right)=\lim _{\longleftarrow} \Gamma \operatorname{Tot} I_{r}^{* *} .
$$

Since $H^{-n}\left(\Gamma \operatorname{Tot} I_{r}\right)=\mathbb{H} C_{n}\langle r\rangle(X)$, we have an exact sequence for each $n$ (see [W, 3.5.8]):

$$
0 \rightarrow \lim ^{1} \mathbb{H} C_{n+1}\langle r\rangle(X) \rightarrow \mathbb{H} C_{n}(X) \rightarrow \lim _{\longleftarrow} \mathbb{H} C_{n}\langle r\rangle(X) \rightarrow 0 .
$$

As $r \rightarrow \infty$ the tower $\left\{\mathbb{H} C_{n}\langle r\rangle(X)\right\}$ stabilizes at $H C_{n}(A)$ as soon as $r>n$ by 2.3 and 2.4.

\section{APPENDIX: HyPERCOHOMOLOGY}

If $K^{*}$ is a cochain complex of sheaves on a site $X$, the hypercohomology of $K^{*}$ is well known - as long as $K^{*}$ is bounded below or $X$ has finite cohomological dimension [EGA, $0_{I I I}$ ]. Unfortunately, that is not the case for many of the complexes considered in this paper. The point of this appendix is to explain what hypercohomology is.

Classical homological algebra uses the following definition of hypercohomology [CE], [EGA, $\left.0_{I I I}\right]$, $[\mathrm{W}, 5.7 .10]$. Because $X$ has enough injective sheaves, we can form an injective Cartan-Eilenberg resolution $I^{* *}$ of $K^{*}$ in the sense of [CE, XVII.1], [EGA, $\left.0_{I I I}(11.4 .2)\right]$ or $[\mathrm{W}, 5.7 .9]$ : there is an augmentation $K^{*} \rightarrow I^{* 0}$ such that the maps on coboundaries and on cohomology are injective resolutions of $B^{*}(K)$ and $H^{*}(K)$. The product total complex Tot $I^{* *}$ is independent of the choice of $I$ up to chain homotopy equivalence [CE, XVII(1.2)], and so is the product total complex $\mathbb{H}\left(X, K^{*}\right)=\Gamma\left(\operatorname{Tot} I^{* *}\right)=\operatorname{Tot} \Gamma\left(I^{* *}\right)$ of the double complex of global sections. We define the Cartan-Eilenberg hypercohomology of $K^{*}$ to be

$$
\mathbb{H}^{i}\left(X, K^{*}\right)=H^{i}\left(\mathbb{H}\left(X, K^{*}\right)=H^{i}\left(\Gamma\left(\operatorname{Tot} I^{* *}\right)\right) .\right.
$$


In this paper we shall use Cartan-Eilenberg hypercohomology. Axiom (0.3) for our cyclic homology theory follows from Thomason's Descent Theorem [T, 1.46] (see [WG, A.5]); if $\{U, V\}$ is a cover of $X$, then there is a Mayer-Vietoris sequence for every complex $K^{*}$ :

$$
\begin{aligned}
\cdots \mathbb{H}^{n}(X, K) & \rightarrow \mathbb{H}^{n}\left(U,\left.K\right|_{U}\right) \oplus \mathbb{H}^{n}\left(V,\left.K\right|_{V}\right) \\
& \rightarrow \mathbb{H}^{n}\left(U \cap V,\left.K\right|_{U \cap V}\right) \rightarrow \mathbb{H}^{n+1}(X, K) \cdots
\end{aligned}
$$

We can interpret this construction in terms of derived categories. A quasiisomorphism $K^{*} \rightarrow L^{*}$ induces a quasi-isomorphism $\mathbb{H}\left(X, K^{*}\right) \rightarrow \mathbb{H}\left(X, L^{*}\right)$ by [WG, A.3]. Therefore the hypercohomology complex induces a well-defined functor $K^{*} \mapsto \mathbb{H}\left(X, K^{*}\right)$ from the derived category $D(X)$ of complexes of sheaves of abelian groups to the derived category $D(\mathbf{A b})$. By $[\mathrm{T}, 1.35], \mathbb{H}(X,-)$ is a morphism of triangulated categories.

Spaltenstein [Sp] and Joyal [Joy1] proved independently that the total right derived functor $\mathbf{R} \Gamma: D(X) \rightarrow D(\mathbf{A b})$ of $\Gamma$ exists, and the hyper-derived cohomology of a complex $K^{*}$ is defined by taking its cohomology:

$$
\mathbb{R}^{i} \Gamma\left(K^{*}\right)=H^{i}\left(\mathbf{R} \Gamma\left(K^{*}\right)\right) .
$$

The universal property of total derived functors yields a unique natural transformation $\eta_{K}: \mathbf{R} \Gamma(K) \rightarrow \mathbb{H}(X, K)$ such that $\Gamma(K) \rightarrow \mathbf{R} \Gamma(K) \rightarrow \mathbb{H}(X, K)$ is the natural augmentation $\Gamma(K) \rightarrow \Gamma \operatorname{Tot}\left(I^{* *}\right)=\mathbb{H}(X, K)$.

If $K^{*}$ is bounded below, or if $X$ has bounded cohomological dimension, it is well known that $\eta_{K}: \mathbf{R} \Gamma(K) \cong \mathbb{H}(X, K)$. However, Example A.5 below shows that $\eta_{K}$ is not always an isomorphism, because $\mathbb{R}^{0} \Gamma(K)$ and $\mathbb{H}^{0}(X, K)$ need not be isomorphic.

Here are some details of the construction of $\mathbf{R} \Gamma$. Call a complex $L^{*}$ (globally) fibrant if for every acyclic complex $A^{*}$ the complex $\operatorname{Hom}\left(A^{*}, L^{*}\right)$ is acyclic. (These complexes are called " $K$-injective" in $[\mathrm{Sp}]$, and "acyclic-local" in [BN, 2.6].) The name comes from Joyal's Theorem [Joy] (see [Jar, 2.7]) that fibrant complexes form part of a "global" closed model structure on $\mathbf{C h}(X)$ in which the cofibrations are the degreewise monomorphisms.

More generally, the definition of a fibrant complex makes sense over any abelian category $\mathcal{A}$. By definition, every acyclic fibrant complex is split exact [W, 2.7.5]. Therefore the (triangulated) category $\mathbf{K}_{f i b}(\mathcal{A})$ of fibrant complexes in $\mathcal{A}$ and chain homotopy classes of maps is its own derived category. When $\mathcal{A}$ is the category of sheaves of abelian groups on a space $X$, we shall write $\mathbf{K}_{f i b}(X)$ for $\mathbf{K}_{f i b}(\mathcal{A})$.

Joyal and Spaltenstein proved that every complex is quasi-isomorphic to a fibrant complex. This implies that $\mathbf{K}_{f i b}(X) \cong D(X)$. (Use [W, 10.5.9].) In particular, we can define $\mathbf{R} \Gamma$ by setting $\mathbf{R} \Gamma\left(K^{*}\right)=\Gamma\left(L^{*}\right)$, where $L^{*}$ is a fibrant complex quasi-isomorphic to $K^{*}$.

The next result shows when "Cartan-Eilenberg" hypercohomology is a hyperderived functor, and clarifies [BN, §2]. Recall that an abelian category is complete if it has (infinite) products, so that Tot of a double complex exists. We say that $\mathcal{A}$ satisfies axiom $\left(A B 4^{*}\right)$ if the product of exact sequences is exact. The category of $R$-modules satisfies $\left(A B 4^{*}\right)$ but (as Example A.5 shows) the category of sheaves on a space $X$ does not. 
Theorem A.3. Let $\mathcal{A}$ be any complete abelian category with enough injectives. Then

(i) The total complex Tot I** of any Cartan-Eilenberg resolution is a fibrant complex.

Now suppose that $\mathcal{A}$ satisfies axiom $\left(A B 4^{*}\right)$. Then

(ii) each map $K^{*} \rightarrow$ Tot $I^{* *}$ is a quasi-isomorphism;

(iii) $D(\mathcal{A})$ is isomorphic to the category $\mathbf{K}_{f i b}(\mathcal{A})$;

(iv) the total derived functors $\mathbf{R} F$ and hyper-derived functors $\mathbb{R}^{i} F=H^{i} \mathbf{R} F$ exist for every additive functor $F: \mathcal{A} \rightarrow \mathcal{B}$. In fact, $\mathbf{R} F\left(K^{*}\right)$ is just $F\left(\right.$ Tot $\left.I^{* *}\right)$.

Proof. Part (ii) is a special case of [W, 3.5.11] — which assumes axiom $\left(A B 4^{*}\right)$ since the cohomology of the tower of brutal truncations of $I^{* *}$ satisfies the MittagLeffler condition. Parts (iii) and (iv) follow from (i), (ii) and [W, 10.5.9], as above. It remains to prove (i).

Let $\tau^{>n} K$ denote the good truncation of $K$ [W, 1.2.7]; it is a quotient of $K$ with $B^{n+1}(K)$ in degree $n$ and 0 in all lower degrees. As with any Cartan-Eilenberg resolution, the good truncations of the rows $I^{* q}$ fit together to form a CartanEilenberg resolution $\tau^{p>n} I^{* *}$ of $\tau^{>n} K^{*}$. Since Tot $I$ is the inverse limit of the tower $\operatorname{Tot} \tau^{p>n} I$, we have

$$
\operatorname{Hom}(A, \operatorname{Tot} I)=\lim _{\longleftarrow} H o m\left(A, \operatorname{Tot} \tau^{p>n} I\right) .
$$

Now suppose that $A^{*}$ is acyclic. As each Tot $\left(\tau^{p>n} I\right)$ is a bounded below injective complex, the usual Comparison Theorem for injective complexes [W, 2.3.7] shows that (a) each $\operatorname{Hom}\left(A\right.$, Tot $\left.\tau^{p>n} I\right)$ is acyclic, and (b) each $H o m\left(A\right.$, Tot $\left.\tau^{p>n} I\right) \rightarrow$ $\operatorname{Hom}\left(A, \operatorname{Tot} \tau^{p>n+1} I\right)$ is onto. These facts combine to show that $\operatorname{Hom}(A$, Tot $I)$ is acyclic.

Remark A.4. Suppose that $K^{*}$ is a complex in which all differentials are zero. Then we can choose a Cartan-Eilenberg resolution $I^{* *}$ in which all the horizontal differentials are zero. If $F: \mathcal{A} \rightarrow \mathbf{A b}$ is additive, then $\operatorname{Tot} F\left(I^{* *}\right)$ is the product of the $F\left(I^{p *}\right)[p]$ and the Cartan-Eilenberg hypercohomology of $F$ is $H^{i}\left(\operatorname{Tot} F\left(I^{* *}\right)\right)=$ $\prod R^{p+i} F\left(K^{-p}\right)$. In particular, when $\mathcal{A}$ is Sheaves $(X)$, then for such a complex we have $\mathbb{H}^{i}\left(X, K^{*}\right)=\prod H^{p+i}\left(X, K^{-p}\right)$.

In the absence of axiom $\left(A B 4^{*}\right)$, the hyper-derived groups $\mathbb{R}^{i} F\left(K^{*}\right)$ will not be this product. Here is an example to show that $\mathbb{R}^{i} \Gamma\left(K^{*}\right)$ can differ from $\mathbb{H}^{i}(X, K)=$ $\prod H^{p+i}\left(X, K^{-p}\right)$.

Example A.5. Let $X$ be the one-point compactification of the disjoint union of the spheres $S^{n}, n=1,2, \ldots$ If we identify each $S^{n}$ with an $n$-sphere of radius $1 / n$ about the origin in Hilbert space, then $X$ is the union of the spheres and the origin. If $\iota_{*}^{n}(\mathbb{Z})$ denotes the extension by zero of the constant sheaf $\mathbb{Z}$ on $S^{n}$, let $K^{*}$ be the negative cochain complex with zero differentials and $K^{n}=\iota_{*}^{|n|}(\mathbb{Z})$ for $n<0$ :

$$
K^{*}: \quad \cdots \stackrel{0}{\rightarrow} \iota_{*}^{3}(\mathbb{Z}) \stackrel{0}{\rightarrow} \iota_{*}^{2}(\mathbb{Z}) \stackrel{0}{\rightarrow} \iota_{*}^{1}(\mathbb{Z}) \rightarrow 0 .
$$

By the previous remark, $\mathbb{H}^{0}(X, K)=\prod_{n=1}^{\infty} H^{n}\left(S^{n}, \mathbb{Z}\right) \neq 0$. Similarly, any neighborhood $U$ of the limit point $x$ contains all but finitely many spheres, so we have $\mathbb{H}^{0}\left(U,\left.K\right|_{U}\right)=\prod H^{n}\left(S^{n} \cap U, \mathbb{Z}\right) \neq 0$ and

$$
\underset{U}{\lim } \mathbb{H}^{0}\left(U,\left.K\right|_{U}\right)=\lim _{p \rightarrow \infty} \prod_{n=p}^{\infty} H^{n}\left(S^{n}, \mathbb{Z}\right) \neq 0 .
$$


On the other hand, if $L$ is a fibrant complex which is quasi-isomorphic to $K$, then

$$
\underset{U}{\lim _{\longrightarrow}} \mathbb{R}^{0} \Gamma\left(\left.K\right|_{U}\right)=\underset{U}{\lim _{\longrightarrow}} H^{0}(\Gamma(U, L))=\mathcal{H}^{0}(L)_{x}=\mathcal{H}^{0}(K)_{x}=0 .
$$

Thus for some $U$ we have $\mathbb{R}^{0} \Gamma\left(\left.K\right|_{U}\right) \neq \mathbb{H}^{0}\left(U,\left.K\right|_{U}\right)$.

\section{ACKNOWLEDGEMENTS}

I would like to express my appreciation to Bob Thomason, who found a mistake in an early version of this paper and reminded me that when we double Čech, a presheaf becomes a sheaf. I would like to thank Joe Lipman for reminding me that products of sheaves are not right exact. And I am grateful to Pierre Deligne, Myles Tierney and Rick Jardine for useful discussions about fibrant complexes of sheaves.

\section{REFERENCES}

[B] C. Beckmann, Relative algebraic K-theory and cyclic homology of schemes, preprint 1992.

[BN] M. Bökstedt and A. Neeman, Homotopy limits in triangulated categories, Compositio Math. 86 (1993), 209-234. MR 94f:18008

[C] A. Connes, Non-commutative differential gometry, Publ. Math. IHES 62 (1985), 257-360. MR 87i: 58162

[CE] H. Cartan and S. Eilenberg, Homological Algebra, Princeton Univ. Press, 1956. MR 17:1040e

[EGA] A. Grothendieck and J. Dieudonné, Eléments de Géométrie Algébrique III (Première Partie), Publ. Math. IHES 11 (1961). MR 29:1209

[GdR] A. Grothendieck, On the de Rham cohomology of algebraic varieties, Publ. Math. IHES 29 (1966), 351-359. MR 33:7343

[Joy] A. Joyal, Letter to A. Grothendieck, 1984.

[Joy1] A. Joyal, personal communication.

[Jar] J.F. Jardine, Simplicial presheaves, J. Pure Applied Alg. 47 (1987), 35-87. MR 88j:18005

[L] J.-L. Loday, Cyclic Homology, Grund. Math. Wissen. Band 301, Springer-Verlag, 1992. MR 94a:19004

[L1] J.-L. Loday, Cyclic homology: a survey, Banach Center Publications, vol. 18 (Warsaw), 1986, pp. 285-307. MR 89e:18023

[Sp] N. Spaltenstein, Resolutions of unbounded complexes, Compositio Math. 65 (1988), 121154. MR 89m:18013

[T] R.W. Thomason, Algebraic K-theory and étale cohomology, Ann. Sci. Éc. Norm. Sup. 18 $4^{e}$ série (1985), 437-552. MR 87k:14016

[W] C.A. Weibel, An introduction to homological algebra, Cambridge University Press, 1994. MR 95f: 18001

[W1] C.A. Weibel, The Hodge filtration and cyclic homology, preprint, 1994.

[WG] C.A. Weibel and S.C. Geller, Étale descent for Hochschild and cyclic homology, Comment. Math. Helv. 66 (1991), 368-388. MR 92e:19006

Department of Mathematics, Rutgers University, New Brunswick, New Jersey 08903 USA

E-mail address: weibel@math.rutgers.edu 